\title{
Chryseobacterium gleum infection in patient with extreme malnutrition and hepatic lesion - case report
}

\author{
DIJANA VARDA BRKIĆ ${ }^{1}$, OZRENKA ZLOPAŠA², BRANKA BEDENIĆ ${ }^{1,3}$, VANDA PLEČKO \\ ${ }^{1}$ Department of Clinical and Molecular Microbiology, Clinical Hospital Centre Zagreb, Kišpatićeva 12, Zagreb, Croatia \\ ${ }^{2}$ Division of Intensive Care Medicine, Department of Internal Medicine, Clinical Hospital Centre Zagreb, Kišpatićeva 12, Zagreb, Croatia \\ ${ }^{3}$ School of Medicine, University of Zagreb, Zagreb, Croatia
}

Corresponding author:

Dijana Varda Brkić

Department of Clinical and Molecular Microbiology, Clinical Hospital Centre Zagreb, Kišpatićeva 12, Zagreb, Croatia

Phone: 01 2367-318

Fax: 012367393

E-mail:dijanavb098@gmail.com

\section{ABSTRACT}

Chrysobacterium gleum is a nonmotile, oxidase positive, non-fermentative unsporulate Gram-negative bacillus, bacteria belonging to the genus Chrysobacterium. Infections caused by Chrysobacterium spp are usually health care associated and the most frequent in immunocompromised patients and neonates.

In this report we present a case of a 35-yearold female patient admitted to the hospital for extreme malnutrition and a hepatic lesion. Chrysobacterium gleum was isolated from tracheal aspirate and blood culture. The strain was identified with Microflex MALDI Biotyper (Bruker Daltonik, Fremont, CA). The patient was successfully treated with piperacillin/tazobactam. Empirical therapy is difficult due to intrinsic resistance to most antimicrobials which are usually effective against Gram-negative bacteria.

Key words: Chrysobacterium gleum, MALDI-TOF, susceptibility pattern, piperacillin/ tazobactam, health care associated infections

\section{INTRODUCTION}

Genus Flavobacterium was identified in 1923. It comprised yellow pigmented, asporogenic Gram-negative bacilli. Since then, it had undergone several classification changes. . In 1994, it was assigned to a new genus Chrysobacterium, belonging to the family Flavobacteriaceae. The species most frequently associated with human infections are: Chrysobacterium meningosepticum, Chrysobacterium indologenes, Chrysobacterium odoratum, Chrysobacterium mutlivorum and Chrysobacterium gleum. (1)

C. gleum is rarely reported in the literature; it is predominantly isolated from various clinical specimens (urine, sputum, wound swab, vaginal swab). In previous reports it was not separated from other species in the genus Chrysobacterium due to insuficient identification or misidentification. (2)

\section{CASE REPORT}

In this report we present a case of a 35-yearold female patient who was initially hospitalized for extreme malnutrition (weight $25.5 \mathrm{~kg}$, height $163 \mathrm{~cm}$, body mass index 9.57) and a hepatic lesion. On the third day of hospitalization she developed a high fever and pneumonia and was transferred to the intensive care unit (ICU). Laboratory analysis revealed leukopenia (L $0.49 \mathrm{x}$ 109) (neutro $75.5 \%$, ly $16.2 \%$, mono $8.1 \%$ ) with elevated CRP (164 ng/L). Respiratory insufficiency deteriorated with the development of septic shock, which required intubation and mechanic ventilation. After obtaining microbiological specimens empiric therapy was initiated with meropenem and linezolid. Klebsiella oxytoca, Staphylococcus aureus and Candida crusei were isolated from tracheal aspirate and thus kaspofungine was added in the therapy. After correction of nutritional status, hepatic function recovered and white blood cell count increased. After 18 days of mechanical ventilation, the patient started to breathe spontaneously. Laboratory results showed the recovery of white blood cells and the decrease of inflammatory parameters.On the 28th day in the ICU the development of pulmonary infiltrates was noticed as well as elevated temperature. C. gleum was isolated from blood culture and tracheal aspirate. Empiric therapy with piperacillin/tazobactam was administered for 12 days. Regression of pulmonary infiltrates and clinical improvement were observed after antimicrobial therapy. On the 42 nd day she was transferred to the gastroenterology department for further correction of nutritional status. The body weight of $29.7 \mathrm{~kg}$ was achieved before the transfer.

\section{CHARACTERIZATION OF ANTIMICRO- BIAL RESISTANCE}

The susceptibility to a wide range of antibiotics was determined by $\mathrm{E}$ test and interpreted according to EUCAST standards for Gram-negative non-fermentative bacteria. MICs of antibiotics active against Gram-positive bacteria were interpreted according to EUCAST guidelines for Staphylococcus.

The strain was resistant to imipenem, meropenem, vancomycin, daptomycin and colistin as shown in Table 1., but it was susceptible to ciprofloxacin, cefepime, ceftazidime, tigecycline and piperacillin / tazobactam.

Combined disk test with meropenem and EDTA was positive, indicating the produc- 
tion of metallo- $\beta$-lactamases (MBL) (3), but negative with clavulanic acid and cephalosporins, indicating the absence of an ESBL (extended-spectrum $\beta$-lactamases). (4)

The transferability of meropenem resistance was tested by conjugation (broth mating method) according to Elwell and Falkow using Escherichia coli A15R-resistant to rifampicin as recipient strain (5). Meropenem resistance was not transferred to E. coli recipient strain.

PCR was performed to detect blaMBL genes (blaVIM, blaIMP, blaGIM, blaSPM) (6), but yielded no PCR products and thus carbapenem resistance is likely to be atributted to the chromosomal metalo- $\beta$ lactamase of the genus Chrysobacterium.

Table 1. Minimum inhibitory concentrations of various antibiotics for C. gleum strain

\begin{tabular}{lll}
\hline $\begin{array}{l}\text { Antimicrobial } \\
\text { agent }\end{array}$ & $\mathbf{m l} \boldsymbol{\mu g} /$ & Interpretation \\
\hline Ceftazidime & 1,5 & Susceptible \\
\hline Cefepime & 0,75 & Susceptible \\
\hline $\begin{array}{l}\text { Piperacillin- } \\
\text { tazobactam }\end{array}$ & 2 & Susceptible \\
\hline Imipenem & 16 & Resistant \\
\hline Meropenem & 32 & Susceptible \\
\hline Ciprofloxacin & 0,5 & Resistant \\
\hline Colistin & $>256$ & Resistant \\
\hline Daptomycin & $>256$ & Resistant \\
\hline Vancomycin & 16 & Resistant \\
\hline Tigecycline & 3 & Resistant \\
\hline
\end{tabular}

\section{DISCUSSION}

In this report we have presented a patient with extreme malnutrition and a hepatic lesion. She became immobile due to extreme cahexia. In the hospital she was provided with the intravascular catheter, uri- nary catheter and endotracheal tubus and was on mechanical ventilation for 18 days. In previous reports $80 \%$ of the patients had polymicrobial infection. In our report, except for C. gleum, C. krusei and K. oxytoca were isolated from sputum and P. aeruginosa from urine. Our patient stayed in the ICU for 28 days and received meropenem and linezolid prior to isolation of C. gleum. C. gleum is an unusual human pathogen. Viroca et al. described early neonatal respiratory infection due to $\mathrm{C}$. gleum in three neonates. (7) Lo et al. performed for the first time a focused study on 15 C. gleum isolates. The strains were epidemiologically unrelated and originated mostly from urine and sputum. (2)

The data on antimicrobial susceptibility of Chryseobacterium spp are limited due to a small number of clinical isolates. Clinical microbiologists usually face two problems: disk diffusion test is not an adequate method for testing Chryseobacterium spp and there are no standardized breakpoints for Chryseobacterium spp in EUCAST guidelines. (8) The choice of an antimicrobial drug for the treatment of Chryseobacterium spp infection is difficult because of its intrinsic chromosomal resistance to many antibiotics used for Gram-negative bacteria. Chryseobacterium spp has intrinsic chromosomal resistance to aminoglycosides, meropenem and imipenem. (9) Our patient was treated with piperacillin/tazobactam. The nutritional status of our patient was also corrected, which improved hepatic function. This is in concordance with the previous report from Bridget at al., who treated a 35-year-old patient with anorexia nervosa by rehydration, electrolyte correction and nutritional support, which also improved hepatic function and decreased the transaminases level. (10) Chryseobacterium spp are the only Gramnegative bacteria susceptible to vancomycin. Vancomycin as monotherapy or combined with other antimicrobials including rifampicin was successful in the treatment of neonatal meningitis. (1)

\section{CONCLUSION}

It is necessary to consider Chryseobacterium spp as causative agent of hospital infections, particularly in patients hospitalized in the ICU for more than 21 days, in those who received broad spectrum antibiotics and in those with indwelling devices. Empiric choice of antimicrobial agent is difficult because of intrinsic resistance to most antibiotics effective against Gram-negative bacteria and very often because of the polymicrobial etiology of infections. Modern methods for identification (MALDI-TOF) should ensure a fast and accurate identification of the strain and an adequate antimicrobial susceptibility testing.

\section{REFERENCES}

1. 8th Mandell GL, Douglas, and Bennett JE. Principles and Practice of Infectious Diseases, Edition. New York Elsevier; 2010: 30223023.

2. Lo HH, Chang SM. Identification, characterization, and biofilm formation of clinical Chryseobacterium gleum isolates. Diagn Microbiol Infect Dis. 2014; 79 (3): 298-302

3. Lee K, Lim YS, Yong D, et al.. Evaluation of the Hodge test and the imipenem-EDTA-double-disk synergy test for differentiating metallo- $\beta$-lactamase-producing isolates of Pseudomonas spp. and Acinetobacter spp. J Clin Microbiol 2003; 41:4623- 4629. 
4. Clinical and Laboratory Standards Institute 2013. Performance Standards for Antimicrobial Susceptibility Testing; 23rd Informational Supplement, M100-S23.Wayne, PA: CLSI

5. Elwell LP, Falkow S. The characterizatio of R plasmids and the detection of plasmid-specified genes. In: Lorian V, ed. Antibiotics in Laboratory Medicine. 2nd edn. Baltimore MD: Williams and Wilkins, 1986: 683-721.

6. Poirel L, Walsh TR, Cuveiller V, et al.Multiplex PCR for detection of acquired carbapenemases genes. Diagn Microbiol Infect Dis 2011; 0:119-125.

7. Virok DP, Abrok M, Szel B, et al. Chryseobacterium gleum - a novel bacterium species detected in neonatal respiratory tract infections. J Matern Fetal Neonatal Med. 2014; 14.

8. European Committee on Antimicrobial Susceptibility Testing (EUCAST). Website with MIC distribution. (http://mic.eucasr.org)/ available from March 2010).

9. Kirby JT, Sader HS, Walsh TR, et al. Antimicrobial Susceptibility and Epidemiology of a Worldwide Collection of Chryseobacterium spp.: Report from the SENTRY Antimicrobial Surveillance Program (1997-2001).

10. Bridet L, Martin JJ, Nuno JL. Acute liver damage and anorexia nervosa: a case report. Turk J Gastroenterol. 2014; 25(2):205-8. 